\title{
Quality assessment of stereoscopic image by 3D structural similarity
}

\author{
Kenny H. B. Voo $^{1}$ • David B. L. Bong 1 (D)
}

Received: 27 June 2016 / Revised: 24 November 2016 / Accepted: 6 January 2017/

Published online: 2 February 2017

(C) Springer Science+Business Media New York 2017

\begin{abstract}
Objective image quality assessment is proposed with the intention to substitute human-rated subjective evaluation by using computational method. Several types of two dimensional (2D) image quality metrics were proposed in the last decade to evaluate the quality of 2D images. When three dimensional (3D) or stereoscopic imaging gradually become popular in different areas of application, new objective quality assessments for 3D images had also been proposed. In this paper, a new method for assessing 3D image quality is proposed. This method is an improvement of the popular 2D structural similarity (SSIM) method with the addition of depth information to measure similarity between distorted and reference 3D images. The proposed method was tested on benchmark 3D image databases to gauge its performance. Experiment results show that predicted quality scores, as calculated from the proposed algorithm, are highly correlated with the corresponding subjective scores from manual evaluation. The significance and effectiveness of the proposed method were also evaluated by comparing its performance to other state-of-the-art 3D quality metrics.
\end{abstract}

Keywords Structural similarity $\cdot$ Stereoscopic images $\cdot$ Image quality assessment

\section{Introduction}

Stereoscopic imaging has been widely used in different areas such as in the entertainment industry which includes video games, 3D images as well as 3D movies. In 2009, the movie "Avatar" had become one of the most successful 3D movies that were ever produced [8].

David B. L. Bong

davidblbong@yahoo.com

Kenny H. B. Voo

kennyvoo1989@gmail.com

1 Faculty of Engineering, Universiti Malaysia Sarawak, 94300 Kota Samarahan, Malaysia 